\title{
Hard-X-Ray-Induced Multistep Ultrafast Dissociation
}

\author{
Oksana Travnikova, ${ }^{1, *}$ Tatiana Marchenko, ${ }^{1}$ Gildas Goldsztejn, ${ }^{1}$ Kari Jänkälä, ${ }^{2}$ Nicolas Sisourat, ${ }^{1}$ \\ Stéphane Carniato, ${ }^{1}$ Renaud Guillemin, ${ }^{1}$ Loïc Journel, ${ }^{1}$ Denis Céolin, ${ }^{3}$ Ralph Püttner, ${ }^{4}$ Hiroshi Iwayama, \\ Eiji Shigemasa, ${ }^{5}$ Maria Novella Piancastelli, ${ }^{1,6}$ and Marc Simon ${ }^{1,3}$ \\ ${ }^{1}$ Sorbonne Universités, UPMC Université Paris 06, CNRS, UMR 7614, \\ Laboratoire de Chimie Physique-Matière et Rayonnement, F-75005 Paris, France \\ ${ }^{2}$ Centre for Molecular Materials Research, University of Oulu, P.O. Box 3000, 90014 Oulu, Finland \\ ${ }^{3}$ Synchrotron SOLEIL, L'Orme des Merisiers, Saint-Aubin, BP 48, F-91192 Gif-sur-Yvette Cedex, France \\ ${ }^{4}$ Institut für Experimentalphysik, Freie Universität Berlin, D-14195 Berlin, Germany \\ ${ }^{5}$ UVSOR Facility, Institute for Molecular Science, Okazaki 444-8585, Japan \\ ${ }^{6}$ Department of Physics and Astronomy, Uppsala University, SE-75120 Uppsala, Sweden
}

(Received 17 December 2015; published 27 May 2016)

\begin{abstract}
Creation of deep core holes with very short ( $\tau \leq 1 \mathrm{fs}$ ) lifetimes triggers a chain of relaxation events leading to extensive nuclear dynamics on a few-femtosecond time scale. Here we demonstrate a general multistep ultrafast dissociation on an example of $\mathrm{HCl}$ following $\mathrm{Cl} 1 s \rightarrow \sigma^{*}$ excitation. Intermediate states with one or multiple holes in the shallower core electron shells are generated in the course of the decay cascades. The repulsive character and large gradients of the potential energy surfaces of these intermediates enable ultrafast fragmentation after the absorption of a hard $\mathrm{x}$-ray photon.
\end{abstract}

DOI: 10.1103/PhysRevLett.116.213001

Absorption of a hard x-ray photon $(>1 \mathrm{keV})$ by a molecule containing elements of the third period or below may lead to the transfer of an electron from a deep shell to an unoccupied valence orbital or to its ejection into the continuum. The photoexcited or photoionized molecules with vacancies in deep core electron shells are highly unstable and short-lived. Their lifetime typically counts only about 1 femtosecond (fs) or a few hundreds of attoseconds [1,2]. These deep core-hole states relax by emitting a photon, an electron, or often several electrons in a cascade process, thus producing multiply charged ions, which fragment rapidly.

Because of those Auger cascades, absorption of just a single hard $\mathrm{x}$-ray photon may lead to the loss of multiple electrons. Thus, for example, $\mathrm{Ar}^{7+}$ and $\mathrm{Cl}^{6+}$ can be produced by exposure of $\mathrm{Ar}$ and $\mathrm{HCl}$ gas to $\sim 3 \mathrm{keV}$ weak-field synchrotron radiation $[3,4]$.

At the same time as electronic relaxation occurs, nuclei begin to move. The phenomenon of the so-called ultrafast dissociation (UFD) is known to compete with Auger decay in neutral highly excited molecular species produced by soft $\mathrm{x}$-ray absorption, when an electron from a shallow inner shell is promoted to a strongly antibonding unoccupied valence orbital [5-15]. The typical lifetimes of the states with shallow inner-shell vacancies (e.g., $\mathrm{Br} 3 d$ [5], O $1 s$ [11,12], S $2 p$ $[14,15], \mathrm{Cl} 2 p$ [7-10]) are of the order of few fs, which is sufficient for the light nuclei to move significantly far from the equilibrium ground state geometry. Then, at the time when the inner-shell vacancy is filled by a valence electron, the molecule may be already dissociated. For deeper core holes, like $\mathrm{Cl} 1 s$, which can be created by hard x-ray radiation, the lifetime is too short to allow for extensive nuclear dynamics prior to electronic relaxation. As a result, even for the $\mathrm{Cl} 1 s \rightarrow \sigma^{*}$ resonance with a strongly repulsive potential energy curve, the $\mathrm{H}-\mathrm{Cl}$ bond can elongate on this time scale by not more than just a few picometers [1]. However, in interpreting such hard x-ray data it is important to consider that creation of deep electron vacancies initiates a cascade of relaxation events that occurs on a time scale of several femtoseconds and allows for dissociation. Signatures of UFD were observed following deep core-electron excitation for the third row hydrides, such as $\mathrm{HCl}$ and $\mathrm{H}_{2} \mathrm{~S}$, in 1998 by Hansen et al. [3] by monitoring the formation of a significant amount of neutral hydrogen (40\% and 30\%, respectively) on resonance. Even much earlier in 1978 [16] a group of very narrow fluorescence lines was observed following $\mathrm{S} 1 s$ core-hole creation in $\mathrm{SF}_{6}$ and $\mathrm{CS}_{2}$, which were attributed to sulfur atomic transitions, but their nature was not understood at that time.

In this Letter, we show that the dominant channels of the first step relaxation processes (both radiative and Auger decays) following deep-core-hole excitation lead to intermediate states which bear 1 or 2 holes in shallower inner shells. The longer lifetime of these intermediate states and strong repulsive character of the corresponding potential energy surfaces allow remarkably very fast nuclear dynamics. As an example, we demonstrate that a strong multistep ultrafast dissociation (MUST UFD) is observed for $\mathrm{HCl}$ following promotion of the $\mathrm{Cl} 1 \mathrm{~s}$ electron to the lowest unoccupied molecular orbital- $\sigma^{*}$. A dominant $K L L$ cascade path is depicted in Fig. 1, where one can see the $\mathrm{H}-\mathrm{Cl}$ bond elongation that begins in the initially produced 


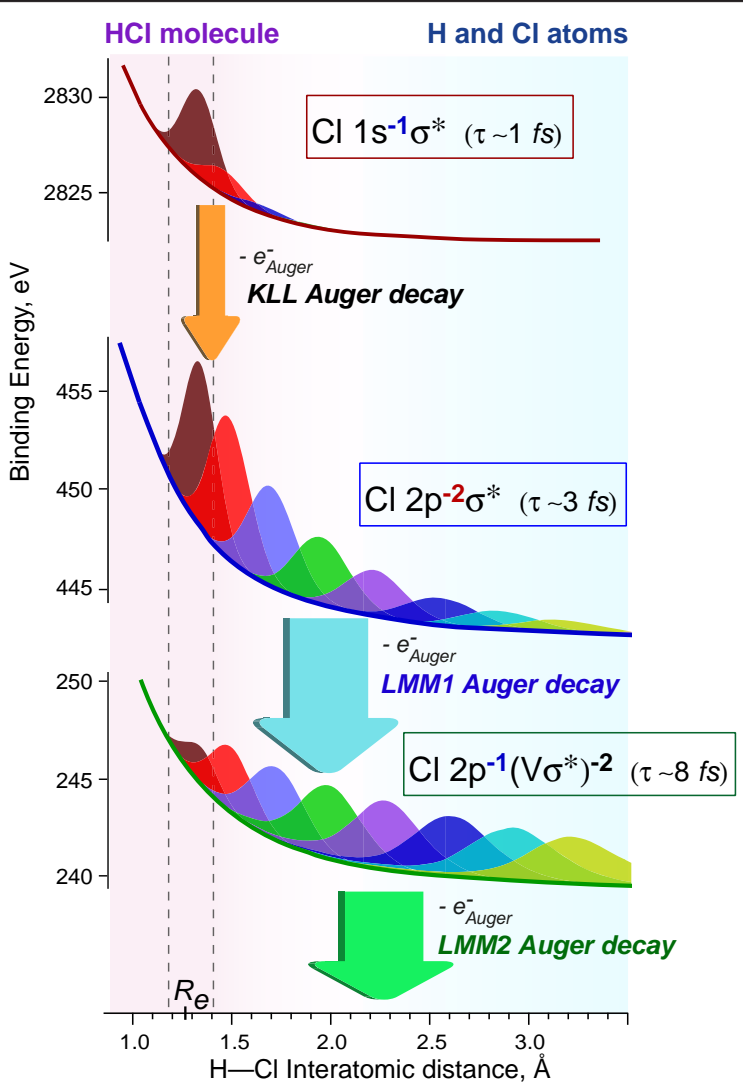

FIG. 1. Potential energy curves for different steps of the $K L L$ Auger cascade following $\mathrm{Cl} 1 s \rightarrow \sigma^{*}$ excitation. Wave-function distributions are shown in different colors for up to 8 fs after X-ray photon absorption with 1 fs increments.

excited state and evolves in every step of the cascade leading to abundant fragmentation. MUST UFD is predicted to be a rather general phenomenon.

The measurements have been performed on the GALAXIES beam line [17] at the $2.75 \mathrm{GeV}$ SOLEIL synchrotron in France using hard $\mathrm{x}$-ray photoelectron spectroscopy (HAXPES) end station [18]. In brief, the beam line delivers linearly polarized light, which is monochromatized by a $\mathrm{Si}(111)$ double crystal and focused by a toroidal mirror. The $\mathrm{HCl}$ sample was commercially obtained from Air Liquide with the purity of $>99 \%$.

The resonant Auger spectra were recorded with a highresolution EW4000 Scienta spectrometer equipped with a wide-angle lens. The spectrometer is installed parallel to the light polarization vector. The electron spectrometer resolution is estimated to be $\sim 150 \mathrm{meV}$ at $100 \mathrm{eV}$ pass energy and the photon bandwidth is $\sim 250 \mathrm{meV}$ around $3 \mathrm{keV}$. Auger decay spectra were recorded at the photon energies corresponding to the top of the $\mathrm{Cl} 1 s \rightarrow \sigma^{*}$ resonance $(\sim 2823.5 \mathrm{eV}[1])$, as well as below the resonance and above the $\mathrm{Cl} 1 s$ ionization threshold (2813 and $3900 \mathrm{eV}$, respectively).

To interpret the experimental results the Auger spectrum of the atomic chlorine was calculated. The excited bound states of the $\mathrm{Cl}$ atom were calculated by using the relativistic GRASP2K package [19] within an average level configuration interaction scheme. The matrix elements for fluorescence and Auger decay (together with continuum wave functions) were calculated using the REOS and AUGER components, respectively, of the RATIP program [20]. The fluorescence decay included dipole contributions only and the Auger decay was calculated within the two-step model. The transition cascades were assumed to start from $1 s \rightarrow 3 p$ excited $\mathrm{Cl}$. The atomic states were constructed as linear combinations of configuration state functions (CSFs) of the same parity and angular momentum. The CSF spaces at each step were constructed from all combinations of $2 p^{m}(3 s 3 p)^{n}$ configurations, where $m$ and $n$ are defined by the ionic state.

Furthermore, bond elongation during the Auger cascades was simulated using a semiclassical approach [21]. In brief, classical trajectories reproducing the phase-space distribution of the vibrational ground level of $\mathrm{HCl}$ are started on the $\mathrm{Cl} 1 s^{-1} \sigma^{*}$ core-excited potential energy curve (PEC). The trajectories can then hop from one state to another according to the corresponding decay widths. The $\mathrm{Cl} 1 s^{-1} \sigma^{*}, 2 p^{-2} \sigma^{*}, 2 p^{-1}\left(V \sigma^{*}\right)^{-2}$ core-hole lifetimes were fixed at $1 \mathrm{fs}$ [1], 3 fs (see below), and $8 \mathrm{fs}$ [22], respectively. Statistical partial widths $\left(\Gamma_{i}\right)$ were assumed at each step of the decay: $\Gamma_{i}=g_{i}\left[\left(\sum_{k} \Gamma_{k}\right) /\left(\sum_{k} g_{k}\right)\right]$, where $g_{i}$ is the degeneracy of the state $i$ (spin and spacial symmetry) and $k$ is the total number of states reached after the decay. In total 60000 trajectories were calculated, which included $1 s^{-1} \sigma^{*}$, $92 p^{-2} \sigma^{*}$, and $842 p^{-1}\left(V \sigma^{*}\right)^{-2}$ states. All PECs were computed at the CASSCF $(13,18)$ level as implemented in MOLPRO [23] with an aug-cc-pcvdz basis set [24,25]. Relativistic corrections were included using the DouglasKroll Hamiltonian [26,27].

The decay of such a deep core hole is very complex, and occurs via a series of subsequent relaxation steps. In Fig. 2 we show a diagram of the dominant radiative and nonradiative decay processes. The recorded $L V V$ spectra, which is a second step in the relaxation decay cascades, are presented in Fig. 3.

In the $\mathrm{HCl}$ molecule, the leading relaxation decays of the $\mathrm{Cl} 1 s^{-1} \sigma^{*}$ state are Auger $K L L$ (76\% [28]), KLM (13\% [28]), and radiative $K L$ (or $K \alpha)(10 \%$ [4,29]) channels. In this Letter we discuss mainly $K L L$ and $K L$ channels, which create $\mathrm{Cl} 2 p^{-2} \sigma^{*}$ and $\mathrm{Cl} 2 p^{-1} \sigma^{*}$ intermediate states, respectively. The latter can be created by direct soft x-ray absorption and have been extensively studied [7-9]. They are known to undergo UFD within the $\mathrm{Cl} 2 p^{-1}$ lifetime of $\sim 8$ fs [22]. The former double-core-hole $\mathrm{Cl} 2 p^{-2} \sigma^{*}$ states are yet exotic and can be also created as so-called "super"shake-up satellites of direct $2 p^{-2}$ double-core-hole ionization [30-32]. The lifetimes of the double-core-hole states $\left(\tau_{\mathrm{DCH}}\right)$ are predicted to be more than two times shorter than that of corresponding single-core-hole states $\left(\tau_{\mathrm{SCH}}\right)$ [33]. The measured $\tau_{\mathrm{SCH}} / \tau_{\mathrm{DCH}}$ ratios for $\mathrm{Ne} 1 s$ and $\mathrm{Ar} 2 p$ core 


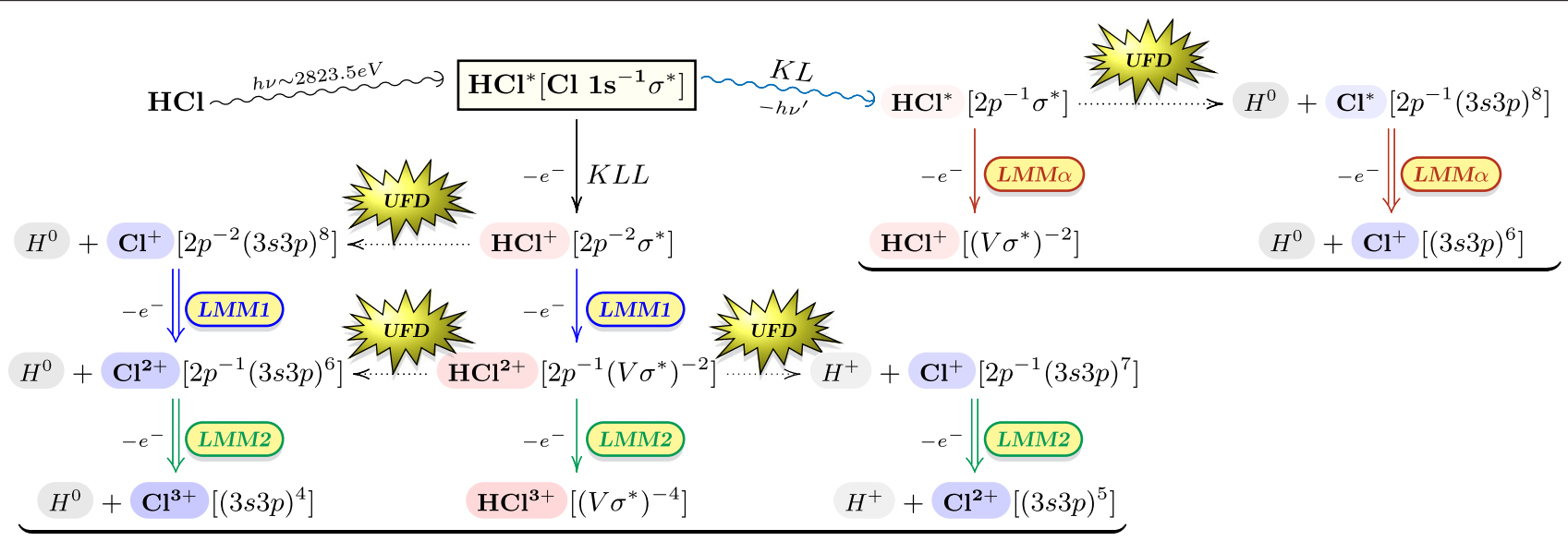

FIG. 2. Diagram of the $L M M$ Auger decays following $K L L$ - and $K L$-cascade paths in $\mathrm{Cl} 1 s^{-1} \sigma^{*}$ core-excited $\mathrm{HCl}$ molecule. Single and double arrows correspond to molecular and atomic Auger decays, respectively.

holes are 2.9 $( \pm 0.1)[34]$ and $2.8( \pm 0.2)[35]$, respectively. By analogy with $\operatorname{Ar} 2 p$ core holes, we predict the $\mathrm{Cl} 2 p^{-2}$ lifetime in $\mathrm{HCl}$ to be about 2.8 times shorter than that of the single-core-hole state $\left(\tau_{\mathrm{DCH}, \mathrm{HCl}} \sim 2.7 \mathrm{fs}\right.$, considering the average lifetime width for $\mathrm{Cl} 2 p^{-1}$ states of $\mathrm{HCl}$ to be $86 \mathrm{meV}$ [22]).

The measured $L V V$ Auger decay spectrum of $\mathrm{HCl}$ (Fig. 3) following $\mathrm{Cl} 1 s \rightarrow \sigma^{*}$ excitation can be divided into three main regions. (I) The kinetic energy region between $187-210 \mathrm{eV}$ is exclusively formed by $L M M 1$ hypersatellite lines, i.e., the Auger decays of the $\mathrm{Cl} 2 p^{-2} \sigma^{*}$ states, produced by the first step $K L L$ Auger decay (see Fig. 2). (II) The $175-185 \mathrm{eV}$ region is dominated by $L M M \alpha$ Auger decay, i.e., the Auger decay of the $\mathrm{Cl} 2 p^{-1} \sigma^{*}$ states after radiative $K L$ relaxation, and contains contributions from the $K L M$ decay path as well as few low intensity $L M M 1$ transitions with final state configurations having one hole in a nonbonding $\sigma$ valence orbital, which is constituted from $\mathrm{Cl} 3 s$ atomic orbitals. (III) The $155-165 \mathrm{eV}$ region contains mainly $L M M 2$ Auger decay transitions, i.e., the Auger decays of the $\mathrm{Cl} 2 p^{-1}\left(V \sigma^{*}\right)^{-2}$ states reached by $L M M 1$ transitions. The region of $165-175 \mathrm{eV}$ has

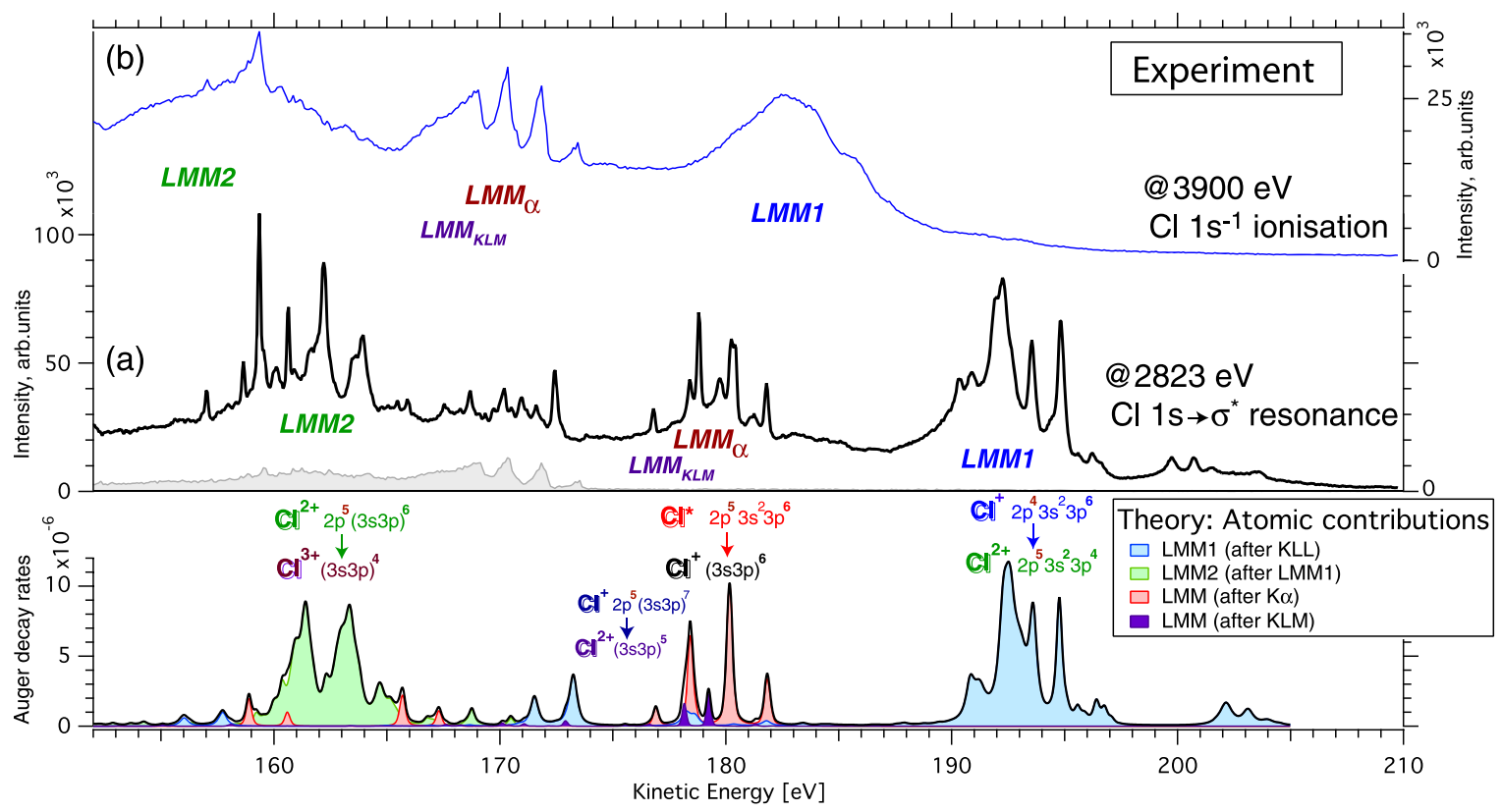

FIG. 3. Top: experimental $L M M$ Auger decay spectra of $\mathrm{HCl}$ recorded after (a) $\mathrm{Cl} 1 \mathrm{~s} \rightarrow \sigma^{*}$ excitation at $2823.5 \mathrm{eV}$ and (b) $\mathrm{Cl} 1 s$ ionization at $3900 \mathrm{eV}$. The gray shaded curve corresponds to the "off"-resonance spectrum recorded at $2813 \mathrm{eV}$. Bottom: Calculated $L M M$ Auger decay spectra following $\mathrm{Cl} 1 s \rightarrow 3 p$ excitation in atomic $\mathrm{Cl}$ (black curve represents a summed total spectrum and individual contribution from different steps of the cascades are shown as filled color curves). All theoretical curves are shifted by -2 eV to match the experimental spectra. 
mixed contributions from $L M M 1,2, \alpha$ Auger decays and is superimposed on the normal Auger decay of $\mathrm{HCl}$ following simultaneous $\mathrm{Cl} 2 p^{-1}$ ionization.

From Fig. 3 one can notice that the intense sharp features are present through the entire $L M M$ spectral region of $\mathrm{HCl}$. In fact, there is a striking similarity of this spectrum with the $L M M$ Auger decay spectrum of Ar following Ar $1 s$ ionization (see, e.g., [36]; Note: core-excited $\mathrm{Cl}$ atom $\mathrm{Cl}$ $\left[1 s^{-1}\right] 3 s^{2} 3 p^{6}$ is valence isoelectronic with Ar). Moreover, the calculated $L M M$ Auger decay spectrum for the atomic $\mathrm{Cl}$ (bottom panel of the Fig. 3) mimics fairly well the $L M M$ spectrum of $\mathrm{HCl}$ even though it does not include molecular decay contributions. There is a very good agreement for the hypersatellite $L M M 1$ part. A somewhat poorer reproducibility of the $L M M 2$ part is probably due to unaccounted electron correlation effects.

Atomiclike decay, which dominates all LMM channels of the $\mathrm{HCl}$ molecule following $\mathrm{Cl} 1 s \rightarrow \sigma^{*}$ excitation, indicates that UFD is present in every LMM step before the next electronic relaxation takes place, including the hypersatellite of the $\mathrm{Cl} 2 p^{-2} \sigma^{*}$ double-core-hole state, whose lifetime is less than 3 fs (Fig. 2). Molecular decays are expected to be broad and form the structureless background [7-10].

The calculated gradient of the PEC for the $\mathrm{Cl} 2 p^{-2} \sigma^{*}$ double-core-hole states is about $50 \%$ larger than that for the dissociative single-core-hole $\mathrm{Cl} 2 p^{-1} \sigma^{*}$ and $1 s^{-1} \sigma^{*}$ states $(\delta E / \delta R \approx-14.5,-10.5,-10.2 \mathrm{eV} / \AA$, respectively) favoring even faster nuclear dynamics than that in $\mathrm{Cl} 2 p^{-1} \sigma^{*}$, which were previously studied using soft $\mathrm{x}$-ray photons $[7,9]$. The calculated wave packet propagation dynamics confirm the possibility of UFD in the $2 p^{-2} \sigma^{*}$ doublecore-hole states of $\mathrm{HCl}$ before the LMM1 Auger decay occurs (Fig. 1). The $\mathrm{H}-\mathrm{Cl}$ bond distances beyond twice the equilibrium bond distance of the ground state $\left(2 R_{e}\right)$ are reached in about 6 fs. Remarkably, the bond elongation, occurring during the first step in the $1 s^{-1} \sigma^{*}$ state, increases the fraction of the wave packet propagating over $\geq 2 R_{e}$ on the $2 p^{-2} \sigma^{*}$ PEC in 6 fs by a factor of 2 (not shown here).

The energy gradients and lifetimes of the $\mathrm{Cl} 2 p^{-1}\left(V \sigma^{*}\right)^{-2}$ states at the next step are comparable to or higher than that of the $\mathrm{Cl} 2 p^{-1} \sigma^{*}$ states of $\mathrm{HCl}$. The wave packet then continues to propagate along the dissociative $\mathrm{Cl} 2 p^{-1}\left(V \sigma^{*}\right)^{-2} \mathrm{PEC}$ leading to even more abundant fragmentation. Assuming the bond dissociation limit of $2 R_{e}$, semiclassical calculations predict the total fragmentation rate $\sim 50 \%$ and $41 \%$ in $K L L$ and $K L$ cascades, respectively, following $\mathrm{Cl} 1 s \rightarrow \sigma^{*}$ excitation by hard $\mathrm{x}$ rays. In comparison, direct $\mathrm{Cl} 2 p \rightarrow \sigma^{*}$ excitation by soft $\mathrm{x}$ rays is predicted to lead to $\sim 37 \%$ fragmentation in the $L M M$ decay.

Above $\mathrm{Cl} 1 s$ ionization threshold the sharp atomic lines, and hence UFD, are not observed for the hypersatellite LMM1 Auger decay (kinetic energy region $>175 \mathrm{eV}$, Fig. 3). The $\mathrm{Cl} 1 s^{-1}$ core-ionized state is bound and its PEC is nearly parallel to that of the ground and $\mathrm{Cl} 2 p^{-1}$ core-ionized states. The $\mathrm{Cl} 2 p^{-2}$ double-core-hole state of $\mathrm{HCl}$ is dissociative but the energy gradients around the equilibrium geometry, reached after the nonradiative $K L L$ decay, are more than twice smaller than that of the $\mathrm{Cl}$ $2 p^{-2} \sigma^{*}$ and hence the nuclear dynamics is too slow to allow for UFD. After 3 fs the $\mathrm{H}-\mathrm{Cl}$ bond is elongated by 8 picometers and only $\sim 1 \%$ of the wave packet reaches the distance of $2 R_{e}$ in $10 \mathrm{fs}$. The triply ionized $\mathrm{Cl} 2 p^{-1} \mathrm{~V}^{-2}$ core-hole states formed at the last step of the $K L L$-cascade are predicted to be strongly dissociative $(\delta E / \delta R \sim-9$, $-13 \mathrm{eV} / \AA$, where latter corresponds to the states with 2 holes in valence nonbonding $\pi$ orbital). However, the strong atomic lines are not observed for the LMM2 decay following $\mathrm{Cl} 1 s$ ionization (Fig. 3). This shows the importance of all the preceding steps of the cascade on the overall photo-induced nuclear dynamics. The dissociative deep-core-hole $\mathrm{Cl} 1 s^{-1} \sigma^{*}$ state with the lifetime of $\sim 1$ fs is impetus to create the domino effect of the bond elongation in the following Auger cascades leading to abundant dissociation on a femtosecond time scale.

In conclusion, we have revealed MUST-UFD phenomenon in the $\mathrm{HCl}$ molecule following deep-core-electron $\mathrm{Cl} 1 \mathrm{~s}$ excitation to the strongly antibonding valence unoccupied $\sigma^{*}$ orbital. MUST UFD leads to pronounced fragmentation at different steps of the following cascade decays. In particular, the intermediate $\mathrm{Cl} 2 p$ double-corehole states of $\mathrm{HCl}$ with the lifetime of $\leq 3$ fs are efficiently produced by the dominant $K L L$ Auger decay channel and dissociate by emitting a neutral hydrogen atom before the next relaxation step occurs. Hence, our results can explain a significant production of neutral hydrogen at the $\mathrm{Cl} 1 s \rightarrow \sigma^{*}$ resonance, previously observed by Hansen et al. [3]. MUST-UFD-like nuclear dynamics, i.e., significant displacement of nuclei from their equilibrium geometry within a few femtosecond time scale, is predicted to be rather general, because the dominant channels of the deep core-hole relaxation processes produce dissociative singleand double-core-hole states as well as multiply charged core-hole states. Recent theoretical studies show that the energy gradients of the double-core-hole $\left(\mathrm{core}^{-2} \mathrm{~V}\right)$ states can be very large. It was calculated to be 3 times larger compared to the core ${ }^{-1} \mathrm{~V}$ state in the case of $\mathrm{H}_{2} \mathrm{O}$ [32]. Therefore, nuclear dynamics is expected to be correspondingly faster in the core ${ }^{-2} \mathrm{~V}$ states.

This work opens a vast horizon for further ultrafast dynamics studies in the course of the cascade processes using hard x-ray spectroscopies. Coincidence energy-resolved measurements of ions and electrons would be advantageous to shed light on the competition between electron and nuclear dynamics in the complex cascade decay paths.

Experiments were performed on the GALAXIES beam line at SOLEIL Synchrotron, France (Proposals No. 20120122 and No. 99150133). We are grateful to SOLEIL staff for smoothly running the facility. N.S. 
acknowledges financial state aid managed by the Agence Nationale de la Recherche, as part of the programme Investissements d'avenir under the reference ANR-11IDEX-0004-02.

* To whom correspondence should be addressed. oksana.travnikova@upmc.fr

[1] M. Simon et al., Phys. Rev. A 73, 020706 (2006).

[2] T. Marchenko et al., J. Chem. Phys. 134, 144308 (2011).

[3] D. L. Hansen et al., Phys. Rev. A 58, 3757 (1998).

[4] R. Guillemin, C. Bomme, T. Marin, L. Journel, T. Marchenko, R. K. Kushawaha, N. Trcera, M. N. Piancastelli, and M. Simon, Phys. Rev. A 84, 063425 (2011).

[5] P. Morin and I. Nenner, Phys. Rev. Lett. 56, 1913 (1986).

[6] E. Pahl, L. S. Cederbaum, H. D. Meyer, and F. Tarantelli, Phys. Rev. Lett. 80, 1865 (1998).

[7] E. Kukk, H. Aksela, O.-P. Sairanen, S. Aksela, A. Kivimäki, E. Nõmmiste, A. Ausmees, A. Kikas, S. J. Osborne, and S. Svensson, J. Chem. Phys. 104, 4475 (1996).

[8] A. Menzel, B. Langer, J. Viefhaus, S. B. Whitfield, and U. Becker, Chem. Phys. Lett. 258, 265 (1996).

[9] O. Björneholm, S. Sundin, S. Svensson, R. R. T. Marinho, A. Naves de Brito, F. Gel'mukhanov, and H. Ågren, Phys. Rev. Lett. 79, 3150 (1997).

[10] O. Travnikova, V. Kimberg, R. Flammini, X.-J. Liu, M. Patanen, C. Nicolas, S. Svensson, and C. Miron, J. Phys. Chem. Lett. 4, 2361 (2013).

[11] O. Björneholm et al., Phys. Rev. Lett. 84, 2826 (2000).

[12] I. Hjelte et al., Chem. Phys. Lett. 334, 151 (2001).

[13] I. Hjelte, M. N. Piancastelli, C. M. Jansson, K. Wiesner, O. Björneholm, M. Bässler, S. L. Sorensen, and S. Svensson, Chem. Phys. Lett. 370, 781 (2003).

[14] K. Le Guen et al., J. Chem. Phys. 127, 114315 (2007).

[15] A. N. de Brito et al., J. Mol. Struct. THEOCHEM 394, 135 (1997).
[16] H. Ågren, J. Nordgren, L. Selander, C. Nordling, and K. Siegbahn, Phys. Scr. 18, 499 (1978).

[17] J.-P. Rueff, J. M. Ablett, D. Céolin, D. Prieur, T. Moreno, V. Balédent, B. Lassalle-Kaiser, J. E. Rault, M. Simon, and A. Shukla, J. Synchrotron Radiat. 22, 175 (2015).

[18] D. Céolin et al., J. Electron Spectrosc. Relat. Phenom. 190, 188 (2013).

[19] P. Jönsson, X. He, C. Froese Fischer, and I. P. Grant, Comput. Phys. Commun. 177, 597 (2007).

[20] S. Fritzsche, Comput. Phys. Commun. 183, 1525 (2012).

[21] N. Sisourat, J. Chem. Phys. 139, 074111 (2013).

[22] M. Kivilompolo, A. Kivimäki, M. Jurvansuu, H. Aksela, S. Aksela, and R. F. Fink, J. Phys. B 33, L157 (2000).

[23] H.-J. Werner, P. J. Knowles, G. Knizia, F. R. Manby, and M. Schütz, WIREs Comput. Mol. Sci. 2, 242 (2012).

[24] T. H. Dunning, J. Chem. Phys. 90, 1007 (1989).

[25] D. E. Woon and T. H. Dunning, J. Chem. Phys. 98, 1358 (1993).

[26] M. Reiher and A. Wolf, J. Chem. Phys. 121, 2037 (2004); 121, 10945 (2004).

[27] A. Wolf, M. Reiher, and B. A. Hess, J. Chem. Phys. 117, 9215 (2002).

[28] M. H. Chen, B. Crasemann, and H. Mark, At. Data Nucl. Data Tables 24, 13 (1979).

[29] F. von Busch, J. Doppelfeld, C. Gunther, and E. Hartmann, J. Phys. B 27, 2151 (1994).

[30] F. Penent et al., J. Electron Spectrosc. Relat. Phenom. B 204, 303 (2015).

[31] M. Nakano, P. Selles, P. Lablanquie, Y. Hikosaka, F. Penent, E. Shigemasa, K. Ito, and S. Carniato, Phys. Rev. Lett. 111, 123001 (2013).

[32] S. Carniato et al., J. Chem. Phys. 142, 014307 (2015).

[33] M. H. Chen, Phys. Rev. A 44, 239 (1991).

[34] G. Goldsztejn et al. (to be published).

[35] M. Žitnik et al., Phys. Rev. A 93, 021401(R) (2016).

[36] F. von Busch, U. Kuetgens, J. Doppelfeld, and S. Fritzsche, Phys. Rev. A 59, 2030 (1999). 